\title{
On the sum of squares of the coefficients of Bloch functions
}

\author{
I.R. Kayumov, K.-J. Wirths
}

April 25, 2019

\begin{abstract}
In this article several types of inequalities for weighted sums of the moduli of Taylor coefficients for Bloch functions are proved.
\end{abstract}

\section{Introduction.}

It was one of the most famous items in function theory, when A.Bloch proved in [3] the following theorem

Theorem A. Let $F$ be analytic in the unit disc $\mathbb{D}$ and $F^{\prime}(0)=1$. Let further $B_{F}$ be the supremum of all numbers $r>0$ with the following property. There exist a complex number $a$ and a domain $\Omega \subset \mathbb{D}$ such that $F$ is injective on $\Omega$ and $F(\Omega)=\{z|| z-a \mid<r\}$. The infimum of these numbers $B_{F}$ is positive.

Since then, this infimum has been called Bloch's constant $B$. In other words, the range of any function of the above type covers a schlicht disc with radius $B$. The exact value of Bloch's constant is not known.

In [12, E. Landau proved that it suffices for estimations of $B$ to consider only those functions that satisfy in addition to the above conditions the inequality

$$
\left|F^{\prime}(z)\right| \leq \frac{1}{1-|z|^{2}}, \quad z \in \mathbb{D} .
$$

This was the beginning of research on the class of Bloch functions that are analytic in $\mathbb{D}$ and satisfy the condition

$$
\sup _{z \in \mathbb{D}}\left(1-|z|^{2}\right)\left|F^{\prime}(z)\right|<\infty .
$$

Keywords: Bloch functions, Subordination, Area functional. AMS classification numbers: 30H30, 30B10. 
This class becomes a normed vector space, the so called Bloch space, if it is endowed with the Bloch norm

$$
\|F\|:=|F(0)|+\sup _{z \in \mathbb{D}}\left(1-|z|^{2}\right)\left|F^{\prime}(z)\right| .
$$

Ch. Pommerenke and his co-authors proved a number of important theorems on the Bloch space (compare for example [1] and [13]), among them some theorems on the behaviour of the coefficients $b_{k}, k \rightarrow \infty$, in the expansions

$$
F(z)=\sum_{k=0}^{\infty} b_{k} z^{k},
$$

where $F$ is in the Bloch space.

Another area of research was related more closely to the condition (1). It concerned the class

$$
\mathcal{B}:=\left\{F|| F^{\prime}(z) \mid \leq \frac{1}{1-|z|^{2}}, z \in \mathbb{D}\right\}
$$

and the behaviour of its elements. In [14] the following problem was posed Problem N: (see [14]) If $f$ is analytic in the unit disc $\mathbb{D}$ and

$$
\left|f\left(r e^{i \theta}\right)\right|<\frac{1}{1-r^{2}}
$$

for what positive real numbers $\alpha$, is it true that

$$
\left|f^{\prime}\left(r e^{i \theta}\right)\right|<\frac{\alpha}{\left(1-r^{2}\right)^{2}} ?
$$

It is known that $\alpha=4$ will suffice.

What is the coefficient region for this class?

The first part of this problem was solved in [19] and [2] with different methods. Partial answers to the second question can be found in [19], [4], 6], [16], and [18]. In the following we shall use

Theorem B. (see [19] and [4]) Let $F \in \mathcal{B}$ and $x \in[0,1 / \sqrt{3}]$ such that

$$
\left|b_{1}\right|=\frac{3 \sqrt{3}}{2} x\left(1-x^{2}\right) .
$$

Then this equation and the inequality

$$
\left|b_{2}\right| \leq \frac{3 \sqrt{3}}{4}\left(1-3 x^{2}\right)\left(1-x^{2}\right)
$$

describe the coefficient region $\left\{\left(b_{1}, b_{2}\right) \mid F \in \mathcal{B}\right\}$.

In [4] and [6] an intimate relation between knowledges on this coefficient region of $\mathcal{B}$ and calculations of estimates for Bloch's constant is revealed. 
Another way to get information on the coefficient region of $\mathcal{B}$ consists in the consideration of weighted sums of moduli of Taylor coefficients. One example for an estimate of this type is the use of Parseval's formula (see $\mathrm{f}$.

i. [13])

$$
\sum_{k=1}^{\infty} k^{2}\left|b_{k}\right|^{2} r^{2 k-2}=\frac{1}{2 \pi} \int_{0}^{2 \pi}\left|F^{\prime}\left(r e^{i \theta}\right)\right|^{2} d \theta \leq \frac{1}{\left(1-r^{2}\right)^{2}}, r \in(0,1) .
$$

Another inequality of this type can easily proved using the maximum principle (compare [17]).

Proposition 1. Let $F \in \mathcal{B}$. Then

$$
\sum_{k=n+1}^{\infty} k^{2}\left|b_{k}\right|^{2} r^{2 k-2} \leq \frac{(n+2)^{n+2}}{4 n^{n}} r^{2 n}, \quad 0 \leq r \leq \sqrt{\frac{n}{n+2}}=r_{n} .
$$

In this estimate, equality is attained for

$$
F_{n}^{\prime}(z)=\frac{n+2}{2}\left(\frac{n+2}{n}\right)^{\frac{n}{2}} z^{n} .
$$

Proof. Let us set

$$
\Phi_{n}(z)=\sum_{k=n+1}^{\infty} k^{2}\left|b_{k}\right|^{2} z^{2 k-2} .
$$

From (2) it follows that

$$
\left|\Phi_{n}(z)\right| \leq \frac{1}{\left(1-r^{2}\right)^{2}}=\frac{(n+2)^{n+2}}{4 n^{n}} r^{2 n}, \quad|z|=r=r_{n} .
$$

Consequently, by the maximum principle

$$
\left|\frac{\Phi_{n}(z)}{z^{2 n}}\right| \leq \frac{(n+2)^{n+2}}{4 n^{n}}, \quad|z| \leq r_{n} .
$$

Setting $z=r \leq r_{n}$ we prove Proposition 1 .

Remark 1. If Proposition 1 is compared with (2), it is obvious that in (2) equality is attained for $r=r_{n}$ and $F=F_{n}$. It is an open question whether there exist other values of $r$ such that in (2) occurs equality.

Inequalities of different type for weighted sums can be found in [11.

In the paper [8], another application of such sums was revealed. Namely, it was shown that the function

$$
\Phi(r):=\sup _{F \in \mathcal{B}}\left(1-r^{2}\right) \sum_{k=1}^{\infty} k\left|b_{k}\right| r^{2(k-1)}
$$


is a decreasing function in $r$ and $\Phi(0.4)<0.9$. Moreover, in the cited paper it was demonstrated that

$$
\sigma_{F}^{2} \leq \Phi(r), \quad r \in[0,1),
$$

where

$$
\sigma_{F}^{2}=\limsup _{r \rightarrow 1} \frac{1}{2 \pi|\log (1-r)|)} \int_{|z|=r}|F(z)|^{2}|d z|
$$

is the asymptotic variance of the Bloch function $F$. The asymptotic variance plays an important role in the probabilistic behaviour of the Bloch function $F$. Namely, Makarov's law of the iterated logarithm (see [10], and also [8, [9]) asserts that

$$
\limsup _{r \rightarrow 1} \frac{\left|F^{\prime}\left(r e^{i \theta}\right)\right|}{\sqrt{\log \frac{1}{1-r} \log \log \log \frac{1}{1-r}}} \leq \sup _{F \in \mathcal{B}} \sqrt{\sigma_{F}^{2}} \quad \text { for almost all } \theta \in[0,2 \pi) .
$$

The present paper is dedicated to further estimates that generalize and sharpen some of the above ones.

\section{Statement and proofs of the results.}

Theorem 1 Let $F(z)=\sum_{k=0}^{\infty} b_{k} z^{k} \in \mathcal{B}, b_{1}=a \in(0,1)$,

$$
\begin{gathered}
a=\frac{3 \sqrt{3}}{2} x\left(1-x^{2}\right), \quad x \in(0,1 / \sqrt{3}), \\
0<r \leq \frac{\sqrt{1 / 3}-x}{1-x \sqrt{1 / 3}} .
\end{gathered}
$$

Then the sharp inequalities

$$
\begin{gathered}
\sum_{k=1}^{\infty} k^{2}\left|b_{k}\right|^{2} r^{2 k} \leq \\
\frac{27 r^{2}\left(1-x^{2}\right)^{2}\left(\left(r^{2}+x^{2}\right)\left(1-r^{2} x^{2}\right)^{2}-6 r^{2} x^{2}\left(1-x^{2}\right)\left(1-r^{2}\right)\right)}{4\left(1-r^{2} x^{2}\right)^{5}},
\end{gathered}
$$

and

$$
\sum_{k=1}^{\infty} k\left|b_{k}\right|^{2} r^{2 k} \leq \frac{27 r^{2}\left(1-x^{2}\right)^{2}\left(3 x^{2}\left(1-r^{2}\right)^{2}+\left(1-x^{2} r^{2}\right)\left(r^{2}-x^{2}\right)\right)}{8\left(1-r^{2} x^{2}\right)^{4}}
$$

are valid. 
Proof. Using the rotation $e^{-i \theta} F\left(e^{i \theta} z\right)$ it is easy to check that

$$
\left\{F^{\prime}(z)|| z \mid=r, F \text { as above }\right\}=\left\{F^{\prime}(r) \mid F \text { as above }\right\} .
$$

Following [4], Satz 2.2.1, this set is given by the bounded region whose boundary is the Jordan curve

$$
\left\{G^{\prime}\left(r e^{i \phi}\right) \mid \phi \in[0,2 \pi]\right\}
$$

where

$$
\begin{gathered}
G^{\prime}(z)=-\frac{a}{x} \frac{z-x}{(1-z x)^{3}}=\sum_{k=1}^{\infty} k A_{k} z^{k-1} \\
=\frac{a}{2} \sum_{k=1}^{\infty} k x^{k-3}\left(2 x^{2}+(k-1)\left(x^{2}-1\right)\right) z^{k-1} .
\end{gathered}
$$

From [4], Lemma 2.3.3 and Lemma 2.3.5 it follows that $G^{\prime}(r z)$ maps the closed unit disc $\overline{\mathrm{D}}$ univalently. Since $F^{\prime}(0)=G^{\prime}(0)$ and $F^{\prime}(r \overline{\mathrm{D}}) \subset G^{\prime}(r \overline{\mathrm{D}})$, this means that $F^{\prime}(r z)$ is subordinate to $G^{\prime}(r z)$. According to a famous Lemma of Rogosinski (see [15]), this implies

$$
\sum_{k=1}^{n} k^{2}\left|b_{k}\right|^{2} \rho^{2(k-1)} \leq \sum_{k=1}^{n} k^{2}\left|A_{k}\right|^{2} \rho^{2(k-1)}, \rho \in[0, r] .
$$

The limiting process $n \rightarrow \infty$ and a straightforward calculation delivers (4). If we let $u=\rho^{2}$ and integrate this inequality with respect to $u$ from 0 to $r^{2}$, we get the assertion (5).

Remark 2. If we assume $F^{\prime}(0)=\epsilon a,|\epsilon|=1$, we may apply the above reasoning to $\bar{\epsilon} F(z)$ and $\bar{\epsilon} F_{x}(z)$ and we see that (44) and (5) are valid likewise.

Remark 3. Another subordination theorem for Bloch functions may be found in [5].

Theorem 2 Let $F \in \mathcal{B}$. Then the sharp inequality

$$
\sum_{k=1}^{\infty} k^{2}\left|b_{k}\right|^{2} r^{2 k} \leq \frac{27}{4} r^{4}, \quad \sqrt{4 / 15} \leq r \leq \sqrt{1 / 3},
$$

is valid.

Proof. If we let $r=\frac{1}{\sqrt{3}}$ in (2), we get

$$
\sum_{k=3}^{\infty} k^{2}\left|b_{k}\right|^{2} \frac{1}{3^{k-1}} \leq \frac{9}{4}-\left|b_{1}\right|^{2}-(4 / 3)\left|b_{2}\right|^{2} .
$$


According to Remark 1, this inequality is sharp. From here we see that

$$
\sum_{k=3}^{\infty} k^{2}\left|b_{k}\right|^{2} r^{2(k-1)} \leq 9 r^{4}\left(\frac{9}{4}-\left|b_{1}\right|^{2}-(4 / 3)\left|b_{2}\right|^{2}\right) .
$$

and consequently

$$
\begin{aligned}
& \sum_{k=1}^{\infty} k^{2}\left|b_{k}\right|^{2} r^{2(k-1)} \leq\left|b_{1}\right|^{2}+4\left|b_{2}\right|^{2} r^{2}+9 r^{4}\left(\frac{9}{4}-\left|b_{1}\right|^{2}-(4 / 3)\left|b_{2}\right|^{2}\right) \\
& =\left(1-9 r^{4}\right)\left|b_{1}\right|^{2}+\left(4 r^{2}-12 r^{4}\right)\left|b_{2}\right|^{2}+\frac{81 r^{4}}{4} \leq \frac{27 r^{2}}{4}, \quad r \geq \sqrt{4 / 15} .
\end{aligned}
$$

From Theorem B we know that it is sufficient to verify this inequality in the case when

$$
b_{1}=\frac{3 \sqrt{3}}{2} x\left(1-x^{2}\right), \quad x \in(0,1 / \sqrt{3}),
$$

and

$$
b_{2}=\frac{3 \sqrt{3}}{4}\left(1-x^{2}\right)\left(1-3 x^{2}\right) .
$$

We have

$$
\begin{gathered}
\left(1-9 r^{4}\right)\left|b_{1}\right|^{2}+\left(4 r^{2}-12 r^{4}\right)\left|b_{2}\right|^{2}+\frac{81 r^{4}}{4}-\frac{27 r^{2}}{4}= \\
(27 / 4)\left(1-3 r^{2}\right) x^{2}\left(1-2 x^{2}+x^{4}+r^{2}\left(-5+16 x^{2}-21 x^{4}+9 x^{6}\right)\right) .
\end{gathered}
$$

It remains to show that

$$
1-2 x^{2}+x^{4}+r^{2}\left(-5+16 x^{2}-21 x^{4}+9 x^{6}\right) \leq 0, \quad 1 / \sqrt{3} \geq r \geq \sqrt{4 / 15} .
$$

Obviously, the first term $1-2 x^{2}+x^{4}$ is positive, whereas the second one is negative for $x \in(0,1 / \sqrt{3})$. Hence, it is evidently enough to verify this inequality at $r=\sqrt{4 / 15}$. In this case we have

$1-2 x^{2}+x^{4}+r^{2}\left(-5+16 x^{2}-21 x^{4}+9 x^{6}\right)=(1 / 15)\left(-1+3 x^{2}\right)^{2}\left(-5+4 x^{2}\right) \leq 0$.

Theorem 2 is proved.

Problem 1. Which is the biggest interval $\left[c_{1}, \frac{1}{\sqrt{3}}\right], c_{1}>0$, such that the inequality of Theorem 2 remains valid in this interval ?

If one adds to the inequality (1) the slightly stronger inequality

$$
\left|b_{1}\right|+\left|\sum_{k=2}^{\infty} k b_{k} z^{k-1}\right| \leq \frac{1}{1-|z|^{2}}, \quad|z|=1 / \sqrt{3},
$$

it is possible to improve the length of the interval from Theorem 2 . 
Theorem 3 Suppose that a function $F \in \mathcal{B}$ satisfies (6). Then the sharp inequality

$$
\sum_{k=1}^{\infty} k^{2}\left|b_{k}\right|^{2} r^{2 k} \leq \frac{27}{4} r^{4}, \quad \sqrt{(9-\sqrt{65}) / 6} \leq r \leq \sqrt{1 / 3}
$$

is valid.

Proof. We have

$$
\sum_{k=2}^{\infty} k^{2}\left|b_{k}\right|^{2} \frac{1}{3^{k-1}} \leq\left(\frac{3}{2}-\left|b_{1}\right|\right)^{2}
$$

and hence

$$
\sum_{k=3}^{\infty} k^{2}\left|b_{k}\right|^{2} \frac{1}{3^{k-1}} \leq\left(\frac{3}{2}-\left|b_{1}\right|\right)^{2}-(4 / 3)\left|b_{2}\right|^{2} .
$$

From here we see that

$$
\sum_{k=3}^{\infty} k^{2}\left|b_{k}\right|^{2} r^{2(k-1)} \leq 9 r^{4}\left[\left(\frac{3}{2}-\left|b_{1}\right|\right)^{2}-(4 / 3)\left|b_{2}\right|^{2}\right] .
$$

and consequently

$$
\sum_{k=1}^{\infty} k^{2}\left|b_{k}\right|^{2} r^{2(k-1)} \leq\left|b_{1}\right|^{2}+4\left|b_{2}\right|^{2} r^{2}+9 r^{4}\left[\left(\frac{3}{2}-\left|b_{1}\right|\right)^{2}-(4 / 3)\left|b_{2}\right|^{2}\right] \leq \frac{27 r^{2}}{4} .
$$

In view of Theorem B it is enough to verify this inequality in the case when

$$
b_{1}=\frac{3 \sqrt{3}}{2} x\left(1-x^{2}\right), \quad x \in(0,1 / \sqrt{3}),
$$

and

$$
b_{2}=\frac{3 \sqrt{3}}{4}\left(1-x^{2}\right)\left(1-3 x^{2}\right) .
$$

Also we may suppose that $r=\sqrt{(9-\sqrt{65}) / 6}$. We have

$$
\begin{gathered}
\left|b_{1}\right|^{2}+4\left|b_{2}\right|^{2} r^{2}+9 r^{4}\left[\left(\frac{3}{2}-\left|b_{1}\right|\right)^{2}-(4 / 3)\left|b_{2}\right|^{2}\right]-\frac{27 r^{2}}{4}= \\
-\frac{9 x}{8}\left(2 \sqrt{3}(73-9 \sqrt{65})+(-737+91 \sqrt{65}) x+2 \sqrt{3}(-73+9 \sqrt{65}) x^{2}+\right. \\
\left.+(1858-230 \sqrt{65}) x^{3}+3(-587+73 \sqrt{65}) x^{5}-72(-8+\sqrt{65}) x^{7}\right)= \\
(1-\sqrt{3} x)^{2}\left(\frac{9}{4} \sqrt{3}(-73+9 \sqrt{65}) x+\frac{9}{8}(-139+17 \sqrt{65}) x^{2}-\frac{9}{8} \sqrt{3}(-153+19 \sqrt{65}) x^{3}-\right.
\end{gathered}
$$




$$
\begin{gathered}
\left.-\frac{9}{8}(-395+49 \sqrt{65}) x^{4}+18 \sqrt{3}(-8+\sqrt{65}) x^{5}+27(-8+\sqrt{65}) x^{6}\right)= \\
(1-\sqrt{3} x)^{2}\left(-1.71348 \ldots x-2.18432 \ldots x^{2}-0.712771 \ldots x^{3}-\right. \\
\left.-0.0569584 \ldots x^{4}+1.941 \ldots x^{5}+1.68096 \ldots x^{6}\right) .
\end{gathered}
$$

From here we see that negative coefficients are dominating. Consequently, this polynomial is negative in the interval $(0,1 / \sqrt{3})$. Theorem 3 is proved.

Problem 2. Which is the biggest interval $\left[c_{2}, \frac{1}{\sqrt{3}}\right], c_{2}>0$, such that the inequality of Theorem 3 remains valid in this interval ?

Now we are going to study the behavior of the area functional

$$
\sum_{k=1}^{\infty} k\left|b_{k}\right|^{2} r^{2 k}
$$

A simple integration of the inequality (2) gives us the inequality

$$
\sum_{k=1}^{\infty} k\left|b_{k}\right|^{2} r^{2 k} \leq \frac{r^{2}}{1-r^{2}}
$$

which is, unfortunately, not sharp for all $r \in(0,1)$.

Theorem 4 Let $F \in \mathcal{B}$ be as in Theorem 1 and let $r \leq \frac{1}{\sqrt{3}}$.

Then for any $n \in \mathbb{N} \backslash\{1\}$ the inequality

$$
\sum_{k=2}^{n} k\left|b_{k}\right|^{2} r^{2 k} \leq \frac{3\left(9-4 a^{2}\right)^{2}}{64 a^{4}} \sum_{k=2}^{n} \frac{1}{k}\left(\frac{4}{3} a^{2} r^{2}\right)^{k}
$$

is valid.

Proof. Since $\left|F^{\prime}(z)\right| \leq \frac{1}{1-|z|^{2}} \leq \frac{3}{2}$ for $r \leq \frac{1}{\sqrt{3}}$, the image of the unit disc under $F^{\prime}(z / \sqrt{3})$ lies in the disc with radius $3 / 2$ around the origin. Hence, this function is subordinate to the function

$$
H(z)=\frac{3}{2} \frac{z+\frac{2 a}{3}}{1+\frac{2 a}{3} z}
$$

As

$$
F^{\prime}\left(\frac{z}{\sqrt{3}}\right)=a+\sum_{k=2}^{\infty} k b_{k}\left(\frac{z}{\sqrt{3}}\right)^{k-1}
$$

and

$$
H(z)=a+\sum_{k=2}^{\infty} \frac{3}{2} \frac{9-4 a^{2}}{9}\left(-\frac{2 a}{3}\right)^{k-2} z^{k-1}
$$


we get from Rogosinski's theorem (see again [15])

$$
\sum_{k=2}^{n} k^{2}\left|b_{k}\right|^{2} \frac{1}{3^{k-1}} \leq \frac{9\left(9-4 a^{2}\right)^{2}}{64 a^{4}} \sum_{k=2}^{n}\left(\frac{4 a^{2}}{9}\right)^{k} .
$$

Now we summands of both sums by $\lambda_{k}=\frac{\left(3 r^{2}\right)^{k}}{k}$. Since this is a decreasing sequence of nonegative numbers, using properties of the Abel transformation (see also [7, Theorem 6.3), we get

$$
3 \sum_{k=2}^{n} k\left|b_{k}\right|^{2} r^{2 k} \leq \frac{9\left(9-4 a^{2}\right)^{2}}{64 a^{4}} \sum_{k=2}^{n}\left(\frac{4 a^{2}}{3}\right)^{k} \frac{r^{2 k}}{k} .
$$

This results in the inequality (7).

Corollary 1. The limiting process $n \rightarrow \infty$ in this theorem results in the inequality

$$
\sum_{k=2}^{\infty} k\left|b_{k}\right|^{2} r^{2 k} \leq \frac{3\left(9-4 a^{2}\right)^{2}}{64 a^{4}}\left(-\log \left(1-\frac{4 a^{2} r^{2}}{3}\right)-\frac{4 a^{2} r^{2}}{3}\right)=B_{a}(r),
$$

where $r \leq \frac{1}{\sqrt{3}}$.

An immediate consequence of Corollary 1 is

Corollary 2. For $F \in \mathcal{B}, r \leq \frac{1}{\sqrt{3}}$, the inequality

$$
\sum_{k=2}^{\infty} k\left|b_{k}\right|^{2} r^{2 k} \leq \frac{27 r^{4}}{8}
$$

is valid.

Proof. We have to prove that

$$
B_{a}(r)-\frac{27 r^{4}}{8} \leq 0, \quad r \leq \frac{1}{\sqrt{3}}
$$

To abbreviate the calculations, we let $w=\frac{4 a^{2} r^{2}}{3}$. With this abbreviation the inequality to prove is the following

$$
H_{a}(w)=\left(1-\frac{4 a^{2}}{9}\right)^{2}(-\log (1-w)-w)-\frac{w^{2}}{2} \leq 0, w \in\left[0, \frac{4 a^{2}}{9}\right] .
$$

Since $H_{a}(0)=0, H_{a}^{\prime}(0)=0, H_{a}^{\prime}(w)<0$ for sufficiently small positive values of $w$, and $H^{\prime}$ has only one positive zero, it is sufficient to prove the above 
inequality for $w=\frac{4 a^{2}}{9}$. If we let $v=\frac{4 a^{2}}{9}$, this task reduces to the proof of the inequality

$$
-\log (1-v)-v-\frac{v^{2}}{2(1-v)^{2}} \leq 0, v \in\left[0, \frac{4}{9}\right] .
$$

This proof can be done by elementary calculations.

Remark 4: Corollary 2 follows immediately from the case $n=1$ of Proposition 1 using $k \leq k^{2} / 2$ for $k \geq 2$.

Theorem 5 Let $F \in \mathcal{B}$. For

$$
R=\frac{1}{4 \sqrt{3}} \sqrt{59-\sqrt{2713}} \leq r \leq \frac{1}{\sqrt{3}}
$$

the following sharp inequality holds

$$
\left(1-\left|b_{1}\right|^{2}\right) \sum_{k=1}^{\infty} k\left|b_{k}\right|^{2} r^{2 k} \leq \frac{27}{8} r^{4}
$$

Proof. At first let us remark it is enough to prove the theorem for the cases $r=R$ and $r=\sqrt{1 / 3}$. Indeed, for the analytic function

$$
\Psi(z)=\left(1-\left|b_{1}\right|^{2}\right) \sum_{k=1}^{\infty} k\left|b_{k}\right|^{2} z^{2 k}
$$

we have

$$
\left|\Psi(z) / z^{4}\right| \leq \frac{27}{8}
$$

for $r=R$ and $r=\sqrt{1 / 3}$ and therefore the inequality holds inside the ring $R \leq r \leq \sqrt{1 / 3}$.

It remains to prove the Theorem 5 in the case $r=R$.

We set $r=R$ and consider three cases (we use these there cases due to technical reasons only, probably there exists a shorter proof).

Case 1: $a \leq 3 / 5$. Remember that $a=\frac{3 \sqrt{3}}{2} x\left(1-x^{2}\right)$. From here we see that $x \leq 0.287$ so that $(1 / \sqrt{3}-x) /(1-x / \sqrt{3}) \geq 0.38 \geq R$ and we can apply Theorem 1 . In view of Theorem 1 we shall show that

$$
\left(1-a^{2}\right) \frac{27 R^{2}\left(1-x^{2}\right)^{2}\left(2 x^{2}+R^{2}\left(1+2\left(-3+R^{2}\right) x^{2}+x^{4}\right)\right)}{8\left(1-R^{2} x^{2}\right)^{4}}-(27 / 8) R^{4} \leq 0
$$

for $x \leq 1 / 4$. We have

$$
\left(1-a^{2}\right) \frac{27 R^{2}\left(1-x^{2}\right)^{2}\left(2 x^{2}+R^{2}\left(1+2\left(-3+R^{2}\right) x^{2}+x^{4}\right)\right)}{8\left(1-R^{2} x^{2}\right)^{4}}-(27 / 8) R^{4}=
$$




$$
\begin{gathered}
-\frac{27 R^{2} x^{4}}{32\left(1-R^{2} x^{2}\right)^{4}}\left(24.5695 \ldots-103.49 \ldots x^{2}+159.036 \ldots x^{4}-\right. \\
\left.-99.9262 \ldots x^{6}+16.2316 \ldots x^{8}+3.88886 \ldots x^{10}\right) .
\end{gathered}
$$

From here we see that the positive coefficients are dominating for $x \leq 1 / 4$ and consequently this expression is negative.

Case 2: $3 / 5<a \leq 3 / 4$. In this case from Corollary 1 it follows that

$$
\begin{gathered}
\sum_{k=2}^{\infty} k\left|b_{k}\right|^{2} r^{2 k} \leq \frac{3\left(9-4 a^{2}\right)^{2}}{64 a^{4}} \frac{16 a^{4}}{9}\left(\log \frac{1}{1-r^{2}}-r^{2}\right)= \\
\frac{\left(9-4 a^{2}\right)^{2}}{12}\left(\log \frac{1}{1-r^{2}}-r^{2}\right) .
\end{gathered}
$$

Consequently

$$
\left(1-a^{2}\right) \sum_{k=1}^{\infty} k\left|b_{k}\right|^{2} r^{2 k} \leq\left(1-a^{2}\right)\left(a^{2} r^{2}+\frac{\left(9-4 a^{2}\right)^{2}}{12}\left(\log \frac{1}{1-r^{2}}-r^{2}\right)\right) .
$$

A little calculus shows that maximum of the last expression is attained at the point $a=3 / 4$ and it is less than $27 r^{4} / 8$.

Case 3: $a \geq 3 / 4$.

According to Corollary 2 the following inequality holds

$$
\sum_{k=2}^{\infty} k\left|b_{k}\right|^{2} r^{2 k} \leq \frac{27}{8} r^{4}
$$

From (8) it follows that

$$
\left(1-\left|b_{1}\right|^{2}\right) \sum_{k=1}^{\infty} k\left|b_{k}\right|^{2} r^{2 k} \leq \max _{3 / 4 \leq a \leq 1}\left(1-a^{2}\right)\left(a^{2} r^{2}+\frac{27}{8} r^{4}\right) \leq \frac{27}{8} r^{4} .
$$

Remark 5. Routine and straightforward calculations show that the number $R$ in Theorem 5 cannot be improved.

Problem 4. How can one get inequalities analogous to the above ones in the intervals $\left[r_{n}, r_{n+1}\right], n \geq 1$ ?

Remark 6. Computer experiments suggest that the inequality (3) can be replaced by $r \leq \sqrt{1 / 3}$. If it is so then simple but routine calculations show that the lower bound $r=\sqrt{4 / 15}=0.5163 \ldots$ from Theorem 2 can be replaced by the sharp number $r=\sqrt{\rho}=0.39466 \ldots$ where $\rho$ is the positive root of the equation

$$
-4-y+81 y^{2}+642 y^{3}-564 y^{4}+1188 y^{5}-82 y^{6}-5809 y^{7}+4581 y^{8}=0 .
$$




\section{Acknowledgements}

We thank the referee for his careful reading of our paper and his proposals that helped to ameliorate it. The research of I. Kayumov was funded by the subsidy allocated to Kazan Federal University for the state assignment in the sphere of scientific activities, project No. 1.12878.2018/12.1.

\section{References}

[1] Anderson J. M., Clunie J. and Pommerenke Ch., On Bloch functions and normal functions. J. Reine Angew. Math., 1974, 270, pp. 12-37.

[2] Avhadiev F. G., Kayumov I. R., Estimates for Bloch functions and their generalization, Complex Variables and Elliptic Equations 29 (1996), no. 3, 193-201.

[3] Bloch A., Les théorèmes de M. Valiron sur les fonctions entières, et la théorie de l'uniformisation, Comptes rendus hebdomadaires des séances de l'Académie des Sciences, Paris 1924, 178, pp. 2051-2052; Annales de la Faculté des Sciences de l'Université de Toulouse 1925, 17, pp. 1-22.

[4] Bonk M., Extremalprobleme bei Bloch-Funktionen, PhD Thesis, TU Braunschweig (1988)

[5] Bonk M., Minda D, and Yanagihara H., Distortion theorems for Bloch functions, Pacific J. Math., 1997179 no. 2, pp. 241-262.

[6] Chen H., Gauthier P. M., On Bloch's constant, J. d'Analyse Math., 1996, 69, pp. 275-291.

[7] P. L. Duren, Univalent Functions, Springer, New York (1983)

[8] Ivrii O., Kayumov I. R., Makarov's principle for the unit ball in Bloch space, Mat. Sb. 2017 208, pp. 96-110.

[9] Kayumov I. R., The law of the iterated logarithm for locally univalent functions, Ann. Acad. Sci. Fenn. Math., 200227 no. 2, pp. 357-364.

[10] Makarov N. G., On the distortion of boundary sets under conformal mappings, Proc. London Math. Soc. 51 (1985), 369-384.

[11] Kayumov I.R., A note on an area type functional of Bloch functions, Lobachevskii J. Math. 2017 33, pp. 464-468.

[12] Landau E., Über die Bloch'sche Konstante und zwei verwandte Weltkonstanten, Math. Z. 1929, 30, pp. 608-634. 
[13] Pommerenke Ch., On Bloch functions, J. London Math. Soc. (2), 1970, 2, pp. 689-695.

[14] Research Problems, Bull. Amer. Math. 1965, 71, pp. 855-857.

[15] Rogosinski W., On the coefficients of subordinate functions, Proc. London Math. Soc. 1943, 48, pp. 48-82.

[16] Roth O., A nonlinear extremal problem for Bloch functions with application to geometric function theory, Arch. Math. 2003, 81, pp. 655-665.

[17] Ruscheweyh St., Wirths K.-J., On extreme Bloch functions with prescribed critical points, Math. Z. 1982, 180, pp. 91-105.

[18] Sugawa T., Terada T., A coefficient inequality for Bloch functions with application to uniformly locally univalent functions, Monatshefte Math. 2009, 156, pp. 167-173.

[19] Wirths K.-J., Über holomorphe Functionen, die einer Wachstumsbeschränkung unterliegen. Arch. Math., 1978, 30, N 6, pp. 606-612.

I. R. Kayumov

Kazan Federal University

Kremlevskaya 18

420008 Kazan, Russia

e-mail: ikayumov@kpfu.ru

Karl-Joachim Wirths

Institut für Analysis

TU Braunschweig

38106 Braunschweig

Germany

e-mail: kjwirths@tu-bs.de 